\title{
Correspondence
}

\author{
Editor: Ian Pullen
}

Contents: Comparing the quality of data from several clinical trials/Cerebral ventricular size and dyskinesia/Chronic fatigue syndrome/DASH scale/ Delusional memory in schizophrenia/Biological basis of behaviour/Enhancement of recovery from psychiatric illness by methylfolate/Anorexia nervosa: is in-patient refeeding necessary?/Anorexia nervosa: is conflict hypothesis valid?/Abreaction before ECT/Special medical and nursing care needs of people with severe learning difficulties/ Jaspers' psychopathology/Systemic family therapy/ Polycythaemia and psychotic depression/Phobic disorders and benzodiazepines in the elderly/NMS complicated by diazepam.

\section{Comparing the quality of data from several clinical trials}

SIR: There is an increasing need for satisfactory statistical techniques to compare the results of clinical trials. There has been much debate about the merits and demerits of meta-analysis, the application of which is well illustrated by Souza \& Goodwin (Journal, May 1991, 158, 666-675) in comparing studies with lithium.

Ideally meta-analysis should only be carried out when all trial data are available, and this is seldom the case. Even if these are accessible there are many differences between the designs of trials that make combination of data unsatisfactory. In deciding which studies are good and deserve special attention, and which are less satisfactory, both clinical and statistical methods are important. Chalmers et al (1981) list 30 aspects of trial methods which are differentially scored so that each trial can score a maximum of 100 points. Although this is valuable, some of the items (e.g. appearance and taste of placebo medication, biological measure of compliance) are biased towards drug trials, other trials, apart from randomised control trials, are excluded, and there is less attention paid to the clinical validity of studies.

There is a need for similar measures of quality for studies of all psychiatric treatments. We suggest that 10 features, which satisfy the requirements for both good clinical and statistical methods (Pocock, 1983; Johnson, 1989), are the most important in evaluating and comparing the results of psychiatric treatments. Together this constitutes a Clinical Validity Index (CVI). These are (with main positive qualities in parentheses): representativeness of population studied (clinical subjects, adequate diagnosis); selection of patients (clear acceptance and rejection criteria); mode of allocation (randomised); sample size (prior estimate of numbers required to show difference of clinical value or sufficient numbers recruited to avoid Type II error); compliance (low ( $<20 \%$ ) drop-out rate, monitoring of treatment and analysis of all evaluable patients); bias-free assessments (independent assessors and/or double-blind procedure); appropriate statistical analyses (covering a range of features usually requiring an independent statistician to be involved in the study); interpretation of findings (conclusions justified by data analyses, both negative and positive); and independence of investigators (evidence of open-mindedness of investigators with equal acceptance of positive and negative results).

Most of these features can be derived from examination of published papers without the need for further material; the last is the most difficult to evaluate and possibly the most important. We suggest that if each of these is given a score of one then a total score of seven or more is necessary before a trial result can be accepted as clinically valid (and included in further evaluation such as meta- analysis). Without such additional assessments there is a danger that unnecessary confusion can arise by combining the findings of good and poor clinical trials.

We thank Tony Johnson for helpful comments.

Chalmers, T. C., Smith, H. Jr., Blackburn, B., et al (1981) A method for assessing the quality of a randomized control trial. Controlled Clinical Trials, 2, 31-49.

JoHnson, T. (1989) Methodology of clinical trials in psychiatry. In Research Methods in Psychiatry: a Beginner's Guide (eds C. Freeman \& P. Tyrer), pp. 12-45. London: Gaskell.

Pocock, S. J. (1983) Clinical Trials: a Practical Approach. Chichester: John Wiley.

\section{St Charles Hospital}

Peter Tyrer

London $\mathrm{W10} 6 \mathrm{DZ}$

JONATHAN TYRER

Department of Probability and Statistics

University of Sheffield 